\section{Moving closer to ideal care for heart failure patients using eHealth and Artificial Intelligence}

Heart failure (HF) is a growing challenge which imposes unsustainable Artificial Intelligence (Al) driven eHealth system that enables self-care for patients with chronic HF. Al, personalised coaching and serious gaming a huge potential to transform healthcare from doctor-driven to patient-driven care, reducing clinical burden and costs per patient while improving the quality of care.

\section{eart failure (HF) is a condition
that develops when the heart that develops when the heart
muscle is unable to pump an} dequate supply of blood to meet the body's needs.

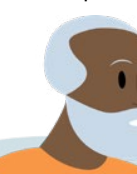

the most prevalent chronic diseases and is estimated to affect approximately 3.6 today. Worryingly, this figure is set to reach more than five million by 2025, survival rates of patients with conditions

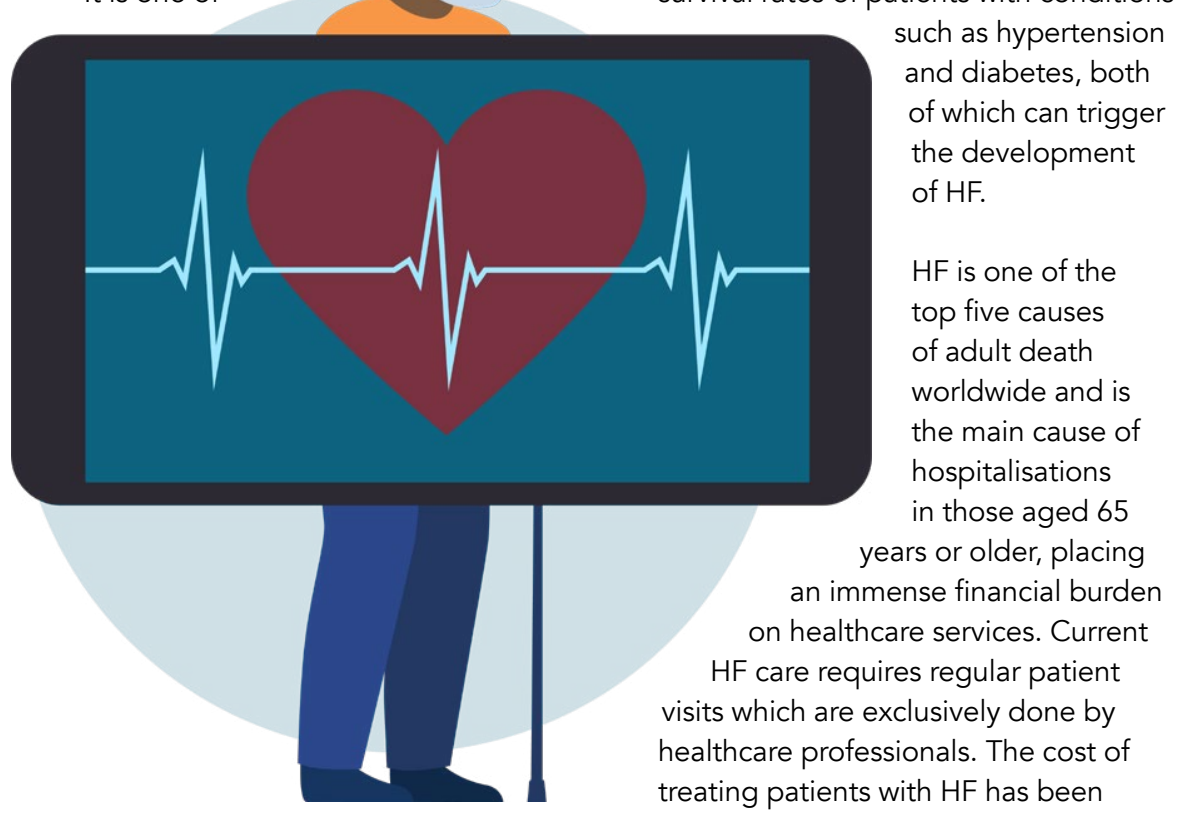
demands on healthcare systems worldwide. Professor Hans-Peter Brunneare leading the international PASSION-HF project, which is developing included, providing true interactive self-management. This approach has million people across north-west Europe stimated as $2 \%$ of the total healthcare expenditure in Europe and North inpatient care. These costs will continue to rise as the prevalence of $H F$ grows. Unfortunately, current healthcare services are not sufficient to meet the suffer from a range of comorbidities. There is also an increasing inequality in the distribution of medical care in rural versus urban regions, and an increasing gap between the growing number of patients and the capacity ( Eventualy, high-quality care will no longer be Wirtable for the entire HF population. Without the active participation of parients in their care processes, the in the current problem facing healthcare systems in terms of treating heart failure of other chronic diseases that impose an enormous financial and societal burden.

Digital technologies have the potential to alleviate much of this burden on healthcare services and improve patien treatment, but eHealth solutions are currently not integrated in the care processes and value chains. HF eHealth products are stand-alone add-ons to standard treatment protocols, rather than being used to support patients PAtient Self-car uSing-Health chrONic Heart Failure e-Health in consortium proposes (ASSON-HF) needs of HF patients who often also patients, it is also applicable to the care

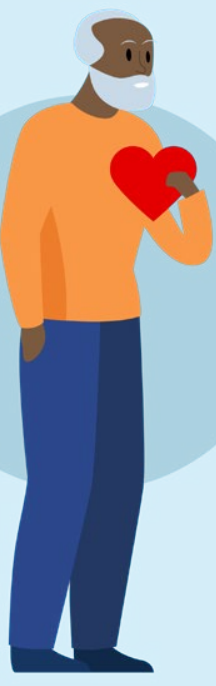

DoctorME welcomes you and asks how you
requested data eg, blood pressure values

care by using digital decision-making on algorithms and Artificial Intelligence (Al) to actively involve patients in their care process. The goal is to move away from a 'one size fits all' approach towards personalised self-managed care. This offers huge potential for reducing clinical burden and the cost of care per patient, as well as improving patient outcomes including reduction of hospitalisations (due to a more personalised treatment of HF) and ensuring access to health avalability of healthcare profess

\section{A NEW VISION OF HEART}

FAILURE CARE

Through an inspiring

clinicians, academics, IT experts, patients, and carers, the PASSIONHF consortium is developing an solution: a virtual

'doctor-at-home' system called 'DoctorME, caring together 24/7'. This eHealth system will provide individualised medical advice directly to patients and their informa caregivers to enable homecare and self-management of chronic HF. The system will implement a predictive

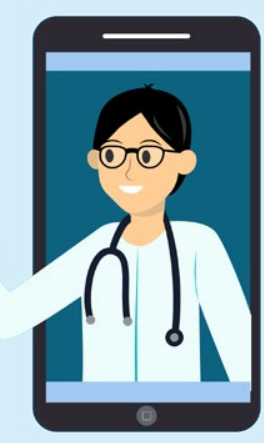

If the therapy can be improved or if there
DoctorME gives you a recommendation.

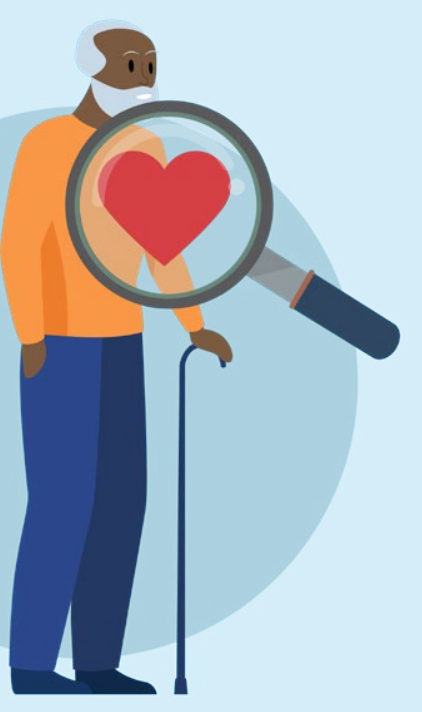

$\mathrm{HF}$ care by including novel features such as a decision-making engine by algorithms based on $\mathrm{HF}$ guidelines, in combination with the integration feedback systems. If, for example, ther are any signs that a patient's health is deteriorating, the eHealth system wil detect this and adapt the medication needs accordingly.

The system also defines processes and decision points where medical professionals need to be included in the process. The integration of seitious gaming motivates regular use of additional diagnostic information.

Without the active participation of patients in their care processes, the burden of HF on our healthcare systems is unsustainable.

Patient independence is maximised through the interactive avatar interface, offering personalised care 24 hours a day, seven days a week. Patients will be empowered with skills to manage their Whe This shoul, more independent way. This should lead to improved regimens which ultimately will have a positive impact on their disease the next to test DoctorME in a pilot project to ddress safety and the proof of concept for patients with $\mathrm{HF}$ and their caregivers.

\section{MOVING AWAY FROM}

DoctomE has the potential to transtorm with $\mathrm{HF}$, wh s shing from doctor-driven patients to provide the enabling a Pum prefession athorion and outpatient clinic visits, and hospitalisations, DoctorME has the potential for significant cost savings

heoretically, up to $50 \%$ of costs associated with HF care could be saved. In addition, the quality of care in HF in real-time assessments and advice via ofvirtue. Moreover, happlication workload for cliniciso educe the to spend more time with complex 


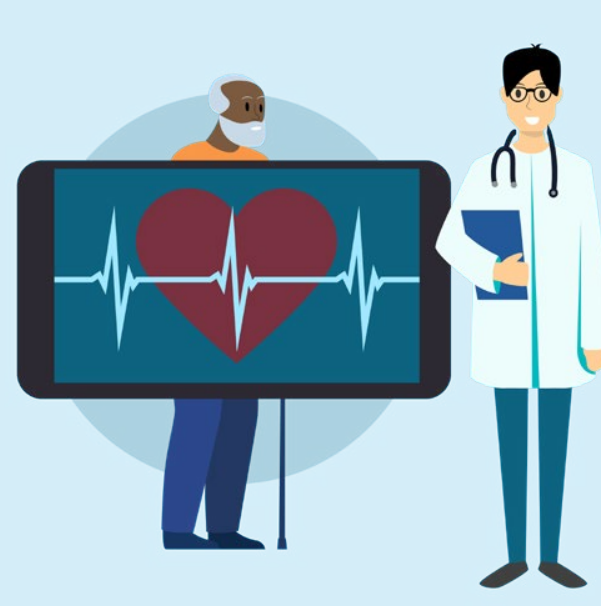

If you feel uncomfortable or have questions, DoctorME
help in the form of counseling or answering questions.

cases and psychosocial aspects of

patient care.

In order to gain the benefits from DoctorME, patients and their caregivers daily care routines. Acceptance of this new technology is therefore a prerequis for its successful implementation. Patients and their caregivers were interviewed to explore their acceptance of eHealth and to gain a better understanding of their preferences for selfThe next-generation eHealth application DoctorME has the potential to transform the day-to-day management of heart demonstrate the feasibility

patients and their cavatar and, overall, to be very receptive towards the idea of using a doctor-at-home.

Key themes were identified in regard to digitalisation of HF care; notably, patients often feel uncertain about their condition and symptoms and require immediate feedback about their current health status, as well as wanting advice on $\mathrm{HF}$ treatmen and medications, including possible side effects. Patients want personalised hour advice from Doctorion and equire it to adapt medication, Irestyle, heir current health stats Patients often failure patients. that can help them remain motivated to achieve this goal. An avatar prototype fulfilling the role of virtual doctor is currently being developed, in which and their caregivers are taken into account to ensure that development is tallored towards their

personalised avatar, it is estimated that $75 \%$ of current visits to a professiona caregiver will be redundant, reducing clinical burden and enhancing patien confidence in self-treatment.

\section{THE LONG-TERM CHALLENGE} The development of this prototype eHealth system by the PASSION HF project is the first step in a new the blueprint for the management various chronic diseases. However it must be acknowledged that the novelty of this next-generation eHeal system may evoke some resistance have to adapt their lifestyle to the needs
of $H F$ and would like an eHealth system healthcare landscape and could become

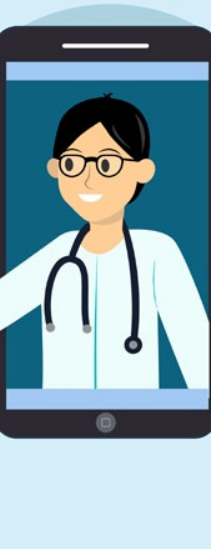

implementation and widespread adoption of the final product, the range of stakeholders such as healthcare providers, insurance analysts, policy makers and patient organisations, amongst others. The interdisciplinary will aid product development by providing advice and guidance, as g the introduction and implementation

of this product in daily care. In order to enhance the of DoctorME, one of the next steps in the will be to demonstrate proof of superiority, as well as cost- and resource saving, in a large clinical tria.

In summary, $\mathrm{HF}$ is a global pandemic currently imposing unsustainable demands in terms of labour and costs on our healthcare system. Using cuttingedge technology, DoctorME, developed in the PASSION-HF project, offers a potential solution by transforming care from solely professional-provided care to Al-enabled, personalised patient self-care with shared responsibilities. Patients wil be empowered with the knowled skills, and tools to manage their condition, thus becoming less dependent
on healthcare professionals.

\section{Behind the Research} (1)

Prof Hans-Peter

Brunner-La Rocca

Hesam Amin MD
E: hesam.amin@mumc.nl T: +31646463783 W: www.doctor-me.eu

Research Objectives

The PASSION-HF consortium uses sophisticated algorithms and Artificial Intelligence to develop personalised self-care for heart failure patients.

\section{Detail}

Address

Maastricht University Medical Center Postbus 5800, 6202 AZ Maastricht The Netherlands

Bio

Professor Hans-Peter Brunner-La Rocca is a cardiologist and vice chairman of the (N) UMC+. He trained in Switzerland, with a focus on heart fallure. His research heart failure; biomarkers and telecoaching play an important role in this.

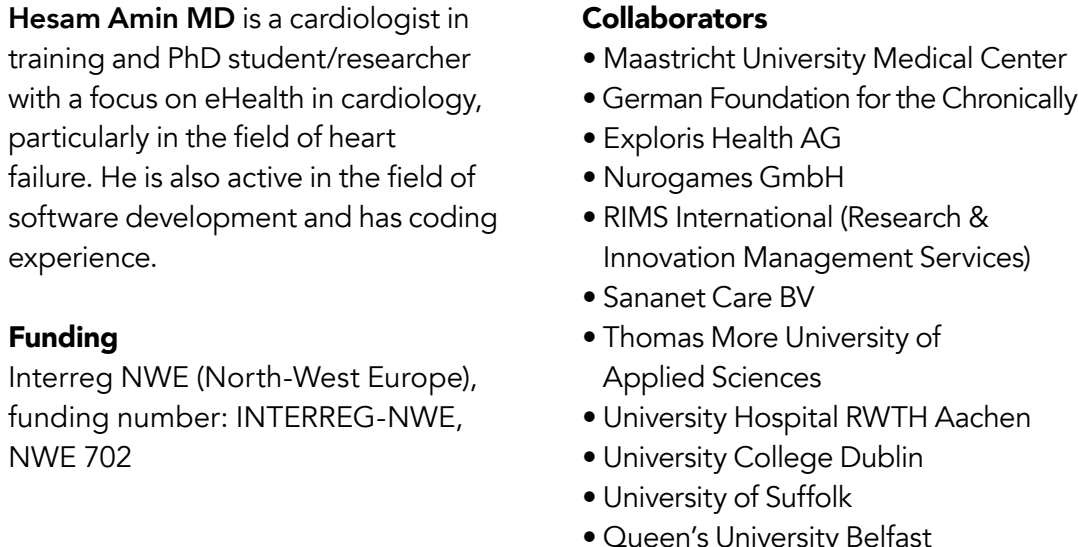
with a focus on eHealth in cardiology particularly in the field of heart failure. He is also active in the field of

Funding

Interreg NWE (North-West Europe), NWE 702 German Foundation for the - Exploris Health AG - Nurogames GmbH - RIMS International (Research \& Innovation Management Services) - Sananet Care BV - Thomas More University of Applied Sciences University Hospital RWTH Aachen - University College Dublin Oreen's University Belfast

\section{References}

Barrett M, Boyne, J, Brandts, J, et al, (2019) Artificia intelligence supported patient self-care in chronic he failure: a paradigm shift from reactive to predictive,

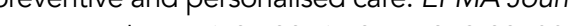

\section{(2) Maastricht UMC+}

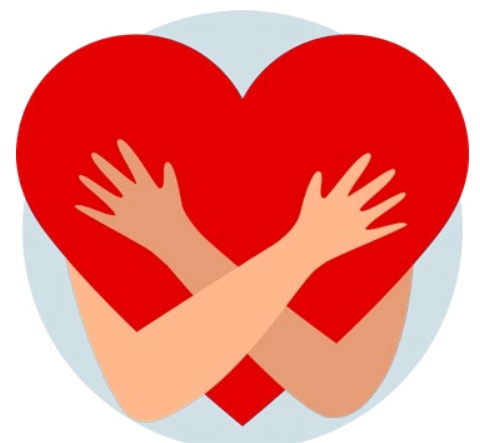

\section{Personal Response}

What hurdles/risks exist that need to be overcome before the widespread adoption of eHealth care systems for the management of not only heart failure, but other chronic diseases?

II One of the first hurdles to overcome is acquiring a seamless connection for data transfer between hospital information systems/electronic health records and eHealth for eHealth is currently stored electronically, it is stored in a way (eg, natural text) that is not easily interpretable for institutes to facilitate safe access to medical data. Once helthcare institutes inte access to medical data. Once

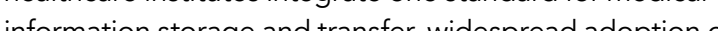
rather than future. systems such as DoctorME. Although information needed eHealth systems like DoctorME will become the standard 